\title{
Trichoderma harzianum T-78 supplementation of compost stimulates the antioxidant defense system in melon plants
}

Running title: Trichoderma inoculated compost enhances antioxidant defenses

Agustina Bernal-Vicente ${ }^{\mathrm{a}}$, José A Pascual ${ }^{\mathrm{a}}$, Fabio Tittarelli ${ }^{\mathrm{b}}$, José A Hernández ${ }^{\mathrm{c}}$, Pedro Diaz-Vivancos, ${ }^{\mathrm{c}, *}$

a Department of Soil and Water Conservation and Organic Waste Management, CEBAS-CSIC, P.O. Box 164, 30100 Espinardo, Murcia (Spain)

b Centro per la Ricerca e la sperimentazione in Agricoltura, Centro di ricerca per lo studio delle relazioni tra pianta e suolo (CRA-RPS), via della Navicella, 2, 00184 Rome, Italy

${ }^{\mathrm{c}}$ Fruit Tree Biotechnology Group, Department of Plant Breeding, CEBAS-CSIC, P.O. Box 164, 30100 Espinardo, Murcia (Spain)

\section{Corresponding author:}

Pedro Diaz-Vivancos (pdv@cebas.csic.es)

Department of Plant Breeding, CEBAS-CSIC, P.O. Box 164, 30100 Murcia (Spain)

Tel. (+34) 968396110

Fax. (+34) 968396213 


\section{ABSTRACT}

BACKGROUND: Compost is emerging as an alternative plant growing media in efforts to achieve a more sustainable agriculture. The addition of specific microorganisms such as Trichoderma harzianum to plant growth substrates increases yields and reduces plant diseases, but the mechanisms of such biostimulants and the biocontrol effects are not yet fully understood. In this work we investigated how the addition of citrus and vineyard composts, either alone or in combination with $T$. harzianum T-78, affects the antioxidant defence system in melon plants under nursery conditions.

RESULTS: Compost application and/or Trichoderma inoculation modulated the antioxidant defence system in melon plants. The combination of citrus compost and Trichoderma showed a biostimulant effect that correlated with an increase in ascorbate recycling enzymes (MDHAR, monodehydroascorbate reductase, DHAR, dehydroascorbate reductase) and peroxidase (POX). Moreover, the inoculation of both composts with Trichoderma increased the activity of antioxidant enzymes, especially those involved in ascorbate recycling.

CONCLUSION: Based on the long-established relationship between ascorbic acid and plant defence responses as well as plant growth and development, it can be suggested that ascorbate recycling activities play a major role in the protection provided by Trichoderma and its biostimulant effect and that these outcomes are linked to increases in antioxidant enzymes. We can conclude that the combination of citrus compost and $T$. harzianum T-78 constitutes a viable, environmentally friendly strategy for improving melon plant production.

Keywords: antioxidant defense system; ascorbate recycling activities; compostgrowing media; Trichoderma harzianum T-78 


\section{INTRODUCTION}

The use of peat as a major constituent of potting media in greenhouse nurseries has been challenged for both economic and environmental reasons. Compost has emerged as an alternative to peat in efforts to achieve a more sustainable agriculture. The following properties make compost desirable for working with plants in greenhouse conditions: low cost of production, high nutrient content and biopesticide properties that would reduce the need for use of chemicals. ${ }^{1}$ Due to the low environmental impact and cost of composting organic waste, such as vineyard and citrus waste, the practice has become an attractive alternative recycling strategy in a great variety of industries. Furthermore, in recent years there has been an increase in the amount of citrus processed by different industries. Citrus composts have shown the ability to stimulate plant growth; a nutriactive effect (the capability of a substrate to express additional and/or synergistic nutritional and biostimulating effects); and biopesticide potential. For example, Bernal-Vicente et $a .^{2}{ }^{2}$ reported that citrus compost partially reduced the impact of Fusarium oxysporum f. sp. melonis and had a biostimulant effect on melon seedlings. Moreover, it has been suggested that enriching compost with specific strains of biological control agents (BCAs) such as Trichoderma spp. can improve plant growth and enhance the efficacy of disease control. ${ }^{3,4}$ Many authors have described the ability of Trichoderma spp to induce local and systemic defense responses that restrict disease development in several economically important agricultural crops. ${ }^{3,5}$ The mechanisms of action of Trichoderma against fungal pathogens include the secretion of antibiotics, competition for space and nutrients, production of lytic enzymes, and induction of host resistance. $^{3}$

Unfavorable environmental conditions such as biotic and abiotic stress exert adverse effects on plant growth and development by inducing a number of metabolic changes, such as the occurrence of oxidative stress due to an enhanced production of reactive oxygen species (ROS) ${ }^{6,7}$ ROS have traditionally been considered as toxic molecules due to their potential to cause oxidative damage to cells. Recent studies, however, have shown that, even under stress situations, ROS play a key role in signal transduction, triggering tolerance and/or acclimatization responses to unfavorable environmental conditions. ${ }^{8}$ The ROS-mediated defense responses include phytoalexin formation, which inhibits the growth of invaders; callose deposition; the strengthening of cell walls; and the synthesis of secondary metabolites and pathogenesis-related (PR) 
proteins. ${ }^{9,10}$ ROS are tightly controlled at both the production and consumption levels by the antioxidative machinery in plants, which includes both enzymatic and nonenzymatic compounds. The main enzymatic arsenal of ROS scavengers plants have to cope with oxidative stress includes enzymes such as peroxidase (POX, EC 1.11.1.7), catalase (CAT, EC 1.11.1.6), superoxide dismutase (SOD, EC 1.15.1.1), glutathione $S$ transferase (GST, EC 2.5.1.18), and the ascorbate-glutathione (ASC-GSH) cycle enzymes [ascorbate peroxidase (APX, EC 1.11.1.11), dehydroascorbate reductase (DHAR, EC 1.8.5.1), monodehydroascorbate reductase (MDHAR, EC 1.6.5.4) and glutathione reductase (GR, EC. 1.6.4.2)].

The antioxidant system protects plants during stress conditions. Antioxidant enzymes are important because of their ability to control ROS metabolism and thereby prevent oxidative damage. How to cope with oxidative damage induced by environmental stresses in an economical and environmentally sustainable way is a major challenge in agricultural practice and research today. ${ }^{10}$ In this regard, compost application could constitute a breakthrough in agriculture by fortifying plant antioxidant defenses. However, little information is available about how compost influences ROS metabolism in plants. ${ }^{10}$

The Trichoderma spp. BCAs have been found to induce accumulation of fungitoxic phenolic compounds. ${ }^{5}$ Moreover, the combination of a mixture of three arbuscular mycorrhizal fungi with compost enhanced drought tolerance in Juniperus oxycedrus L. plants, and this response correlated with an induction of SOD and POX activities. $^{11,12}$ Nevertheless, information regarding the stimulation of the antioxidant system in plants by Trichoderma, alone or in combination with compost, is very scarce. Recently, Mastouri et al. ${ }^{13}$ described that T. harzianum T22 colonization enhanced the antioxidant defense mechanism of tomato plants. These authors reported an increase in the relative expression of genes encoding antioxidant enzymes and correlated the ability of T22-colonized plants to tolerate a wide range of stresses, such as water deficit, salinity and suboptimal temperatures. The colonized plants showed an enhanced redox buffering capacity. ${ }^{13}$

Understanding the nature of Trichoderma-plant interaction as well as the results of combining Trichoderma strains with certain composts that show nutritional and biopesticide effects may provide a potentially useful tool for enhancing crop production. In this work, the effects of T. harzianum T-78 and/or two composts (vineyard and citrus 
waste) on the antioxidant defense system of melon plants were explored. In addition, we also tested as substrate peat supplemented with bentonite, a solid carrier for $T$. harzianum proliferation used for the inoculation of this BCA. ${ }^{14}$

\section{METHODS}

\section{Fungal strains and plant material}

The biological control agent (BCA) T. harzianum T-78, isolated from agricultural soil and deposited in the Spanish Type Culture Collection (CECT 20714), was chosen from the CEBAS-CSIC collection.

Melon seeds (Cucumis melo L. cv. cantasapo) were sown in polystyrene containers and seedlings were grown under standard nursery seedling conditions at Semilleros el Mirador (Murcia, Spain). ${ }^{14}$

\section{Composts}

Vineyard waste compost (C1) was produced by the company Eisercorp (Hellín, Albacete, Spain) as described by Bernal-Vicente et al. ${ }^{15}$ The inoculation of compost C1 with T. harzianum T-78 was performed at the beginning of the maturation phase of the composting process. A non-inoculated compost pile was processed as a control. ${ }^{15}$

Citrus compost (C2) was produced in the Azienda Sperimentale Palazzelli (Lentini), of the CRA-ACM (CT, Italy). C2 composts contained 60\% citrus waste and $40 \%$ green residue, and the specific composting process used was that described by Tittarelli et al. ${ }^{16}$

Physico-chemical and chemical characteristics of the composts and peat used are shown in Table 1. Additional data concerning these characteristics and the nutriactive effect (measured as the increase in fresh weight of melon plants grown in the different substrates with respect to melon plants grown in control peat) of the substrates used have been previously described. ${ }^{2,4}$ Other studies have also addressed the evolution of the T. harzianum T-78 population and the effectiveness of the organic materials used against Fusarium wilt. ${ }^{15}$ 


\section{Experimental design}

The following organic substrates were used in this work: peat; a mixture of peat and bentonite (5\%); and the two above-mentioned composts (C1 and C2). Treatments consisted of non-inoculated and Trichoderma-inoculated substrates. The experiment was carried out using three different polystyrene containers with 10 individual wells per treatment (30 plants per treatment). Each individual container was considered as a replicate within each treatment.

Seeds were germinated in a growth chamber at $28 \pm 1{ }^{\circ} \mathrm{C}$ and $90 \%-95 \%$ relative humidity. After germination the different containers were randomly distributed in a polyethylene-covered greenhouse nursery under natural daylight conditions and were irrigated daily. The temperature and relative humidity during the assay ranged between $25-32^{\circ} \mathrm{C}$ and $40-60 \%$ during the day and between $20-22^{\circ} \mathrm{C}$ and $30-40 \%$ at night. Both temperature and relative humidity were measured with probes and registered in a dataloger (Testo 174H). Plants were sampled at 30 days after sowing (DAS).

Peat and compost C2 were inoculated with a conidial suspension of $T$.

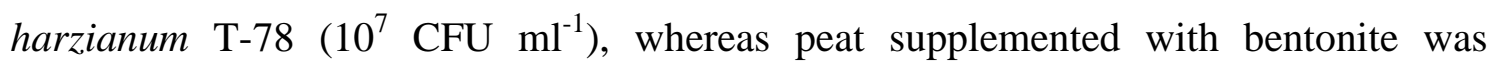
inoculated with immobilized T. harzianum T-78 to achieve a final concentration of $10^{6}$ CFU g ${ }^{-1} .4$ As described above, compost C1 included the BCA in its composition.

\section{Enzymatic assays}

All operations were performed at $0-4{ }^{\circ} \mathrm{C}$. Samples (1-2 g fresh weight) were homogenized with an extraction medium (1/3 w/v) containing $50 \mathrm{mM}$ Tris-acetate buffer (pH 6.0), 0.1 mM EDTA, $2 \mathrm{mM}$ cysteine and 0.2\% (v/v) Triton X-100. For the APX activity, $20 \mathrm{mM}$ sodium ascorbate was added to the extraction buffer. The extracts were filtered through two layers of nylon cloth and centrifuged at $10000 \mathrm{~g}$ for $15 \mathrm{~min}$. The supernatant fraction was filtered on Sephadex G-25 NAP columns equilibrated with the same buffer used for homogenization. For the APX activity, 2 mM sodium ascorbate was added to the equilibration buffer.

The activities of the ASC-GSH cycle enzymes, POX, CAT, and SOD were assayed as described by Diaz-Vivancos et al. ${ }^{6,7}$ Glutathione $S$-transferase (GST) activity was analyzed according to the method of Habing and Jakoby ${ }^{17}$, based on the increase in absorbance at $340 \mathrm{~nm}$ resulting from the formation of a conjugated compound through 
the reaction of GSH with 1-chloro-2,4-dinitrobenzene. Protein was estimated according to the method described by Bradford. ${ }^{18}$

\section{Statistical analysis}

The effects of inoculation with Trichoderma and/or the use of different substrates on the enzymatic antioxidant system in melon plants were tested by a two-way analysis of variance (ANOVA). Analyses were carried out at least in triplicate. Comparisons between treatments were performed using Tukey's test. The statistical procedures were carried out using an SPSS software package.

\section{RESULTS}

The fresh weight (FW) of the melon plants was affected by the substrate used and Trichoderma inoculation as well as by the interaction between both factors (Table 2). When compared with plants grown in non-inoculated peat, the FW of the melon plants grown in the peat, bentonite and compost C1 mixture was slightly lower. No differences were recorded, however, in treatments with compost C2 (Fig. 1). On the other hand, inoculation with T. harzianum T-78 increased the FW of melon plants in all the substrates assayed except for plants grown in inoculated peat, in which a 1.2-fold decrease in FW was observed. The mixture of peat, bentonite and compost C2 showed the best response in terms of FW when T. harzianum T-78 was applied, with a 1.4-fold increase compared to the respective controls. Melon plants grown in inoculated C2 displayed the highest FW value in this study (Fig. 1).

The effect of T. harzianum T-78 inoculation on the antioxidant defense system was studied in the different organic substrates. BAC inoculation and the substrate used, as well as the interaction between both factors, had a significant effect on APX, MDHAR, DHAR and GST activities (Table 2). SOD activity was affected by both factors, but no interaction between the factors was observed. The kind of organic substrate as well as the interaction between substrate and Trichoderma inoculation significantly affected the level of POX. In contrast, CAT activity neither showed significant changes due to the substrate nor to BAC inoculation (Table 2). Finally, GR activity was not detected under our experimental conditions.

In the absence of $T$. harzianum $\mathrm{T}-78$, the use of non-inoculated bentonite on peat had no effect on the antioxidant system, and only a decrease in MDHAR activity was 
recorded when compared with non-inoculated peat (Figs. 2 and 3). Nevertheless, the two composts analyzed affected the levels of enzymatic antioxidants (Figs. 2 and 3). Compost C1 increased the APX, POX and GST activities by up to 1.2, 6.2 and 1.7-fold, respectively, in the leaves of the melon plants studied (Figs. 2A, 3B and 3C). On the other hand, a slight decrease in the ascorbate recycling enzymes (MDHAR and DHAR) was recorded in compost 1 treatments (Figs. 2B and 2C). Compost C2 also significantly stimulated POX and GST levels, by up to 2.3 and 1.8-fold, respectively (Figs. 3B and 3C). Moreover, compost C2 induced a similar decrease in MDHAR to that observed in bentonite and compost C1 treatments (Fig. 2B). SOD activity did not show any significant change in non-inoculated organic substrates (Fig. 3A). Regarding the use of different composts as an alternative to peat, both composts studied clearly stimulated the antioxidant capacity of the melon plants grown on them.

The inoculation of Trichoderma had different effects on the antioxidant system depending on the substrate used (Figs. 2 and 3). Plants grown in Trichodermainoculated peat displayed higher APX and GST activities as well as a decrease in ascorbate recycling enzymes. The inoculated mixture of peat and bentonite, on the other hand, produced a stimulation of GST and ascorbate recycling activities.

The inoculation of compost C1 with Trichoderma increased APX and MDHAR antioxidant activity by up to 1.2-fold, SOD activity by up to 1.5 -fold and POX activity by up to 3.2-fold with respect to the T. harzianum T-78-inoculated peat (Figs. 2 and 3). Interestingly, when inoculated C1 was compared to its control, a decrease in POX and GST activities of about 1.4-fold was observed (Figs. 3B and 3C).

The addition of Trichoderma to compost C2 also induced an increase in ascorbate recycling and POX activities when compared to inoculated peat as well as to the control (non-inoculated C2). Compared to inoculated peat treatments, the MDHAR, DHAR and POX activities in inoculated compost treatments increased by up to 1.2, 1.9 and 2.4-fold, respectively, whereas an increase of about 1.3-fold was recorded with respect to the control (Figs. 2B, 2C and 3B).

\section{DISCUSSION}

The nutriactive and biocontrol effects of the two composts derived from waste generated by vineyards and citrus processing industries (C1 and $\mathrm{C} 2$, respectively) 
showed that these composts, either on their own or in combination with the widely used BCA Trichoderma, contribute to the biological control of $F$. oxysporum in melon., ${ }^{2,45}$ However, the mechanism involved in this response remains to be elucidated, and there is no information available on BCAs and/or composts that enhance plant defense reactions by inducing changes in the antioxidative metabolism. ROS, and hence the antioxidant system, play key roles in many defense processes, including direct antimicrobial action, lignin formation, phytoalexin production, PR protein induction, the hypersensitive response and systemic acquired resistance. ${ }^{4,9}$

The addition of Trichoderma increases the nutriactive effect of all substrates studied except peat, reinforcing the idea that the combination of compost and Trichoderma stimulates plant growth. In this work, the antioxidant defense system was shown to modulate melon plant growth in the composts studied, both in the presence and absence of $T$. harzianum T-78. Regarding the relationship between compost application and stress tolerance, Tartoura and Youssef ${ }^{10}$ have proposed several modes of action to explain how compost improves plant tolerance against adverse environmental conditions. These modes include an increase in the antioxidant capacity of plants coupled with an induction of defense responses such as systemic acquired resistance. These authors observed a correlation between enhanced antioxidant capacity and protection against low temperature-induced oxidative stress. They described that squash plants grown in compost, consisting of a mixture of cow manure, chicken manure and wheat straw, showed significantly higher APX, GR, CAT and SOD activities. ${ }^{10}$ Moreover, a similar increase in ROS-scavenging enzymes has been described in wheat plants grown in higher doses of either municipal solid waste compost or sewage sludge. ${ }^{19}$ Other modes of action through which composts confer protection against unfavorable conditions are related to the physical and chemical properties of the composts. Some of the chemical compounds present in composts can be involved in the regulation of gene and protein expression and be components of enzymatic and nonenzymatic antioxidants, inducing plant tolerance to environmental stress conditions. ${ }^{20}$ Furthermore, compost facilitates the colonization of plant roots by arbuscular mycorrhizal fungi. In fact, the combination of arbuscular mycorrhizal fungi with composted sewage sludge or composted organic residue has been found to induce SOD and POX activities in leaves of Juniperus oxycedrus L. plants. ${ }^{11,12}$ These cited studies have suggested a correlation between the increase in antioxidant enzymes and enhanced 
drought tolerance. In recent works, the overexpression of CuZn-SOD and/or APX in the cytosol of transgenic tobacco plants has been found to alleviate, to some extent, the damage produced by mild water stress conditions and to enhance resistance against Pseudomonas syringae pv. tabaci infection. ${ }^{21,22}$ Under our experimental conditions, we observed an increase in POX and GST activities due to compost application. The POXs are involved in plant defense through different mechanisms such as cell wall stiffening, the production of ROS against attacking organisms and involvement in the synthesis of phytoalexins. ${ }^{23}$ GSTs, on the other hand, have traditionally been associated with the metabolism of xenobiotics and secondary products via the formation of glutathione conjugates, thus protecting cells against oxidative stress. ${ }^{24}$

Trichoderma spp. is a widely used BCA in agriculture due to its beneficial effects in terms of increasing yield and reducing plant diseases. ${ }^{3}$ For example, inoculating tomato seedlings with the $T$. harzianum strain T22 has been found to significantly increase the expression of chloroplastic and cytosolic enzymes belonging to the ASC-GSH cycle (APX, MDHAR, DHAR, and GR) and to produce an increase in $\mathrm{SOD}^{13}$. In addition, according to our results, CAT was not affected by Trichoderma inoculation. ${ }^{13}$ These authors suggest that the enhanced ability of plants to scavenge ROS induced by T22 is related to the tolerance response against different abiotic stresses. Other authors have found that treating sunflower plants with a formulation of $T$. harzianum-NBRI-1055 can induce resistance against the pathogen Rhizoctonia solani by reprogramming oxidant and antioxidant metabolites. ${ }^{25}$

In the present study, we observed that the addition of the T-78 strain to the two composts (C1 and C2) and peat modified the antioxidant capacity of melon plants and the activity of the ascorbate recycling enzymes (MDHAR and DHAR). Yet while the addition of Trichoderma to peat led to a decrease in ascorbate recycling activities, BCA inoculation increased the level of these activities in both composts. Considering that peat is neutral in terms of suppressiveness, the increase in ascorbate recycling enzymes in melon plants by composts inoculated with T. harzianum T-78 could be related to the biocontrol effect of these composts on $F$. oxysporum. ${ }^{2,4,15}$ In this sense, it has been described that the overexpression of MDHAR and DHAR enhanced tolerance to different stress conditions. ${ }^{26,27}$ The authors of these aforementioned studies have shown that increasing the ASC content in plants through enhanced ascorbate recycling can limit damage produced by abiotic stresses. Moreover, increases in ascorbic acid content 
and antioxidant activity have been recently described in Brassica rapa plants treated with T. harzianum TM10. ${ }^{28}$ Ascorbic acid is the most abundant soluble antioxidant in plants. Different studies conducted on ascorbic acid-deficient mutants have highlighted the role this antioxidant plays in plant defense reactions, where it acts as a “crosstalking” signal that coordinates the activity of defense networks complementary to the antioxidant system. ${ }^{29}$ All these cited results suggest that the enhanced tolerance displayed by Trichoderma-colonized plants under abiotic and biotic stress conditions is at least partly orchestrated by the stimulation of the antioxidant defense system, ${ }^{13}$ in which the ascorbate recycling enzymes play a major role. A high capacity to scavenge ROS and recycle oxidized ascorbate and glutathione is in fact an expected mechanism in plants for coping with environmental constraints.

According to previous results, the addition of Trichoderma to compost C2 stimulates plant growth, ${ }^{2,4,15}$ as well as the activity of some antioxidant enzymes such as those involved in ascorbate recycling and POX activity. Among the many key roles that ascorbate plays in plants biology, its involvement in plant growth, influencing mitosis and cell growth, has been widely described. ${ }^{30}$ Moreover, ascorbate could enhance cell wall plasticity by affecting the activity of the POXs responsible for cell wall stiffening. ${ }^{31}$ In addition, there are several lines of evidence indicating that plant POXs are involved in cell wall metabolism and development. In particular, POXs are responsible for the stiffening of the cell wall and lignin and suberin deposition. ${ }^{31}$ The increase in fresh weight induced in Trichoderma-inoculated C2 could be therefore be related to an enhanced antioxidant capacity. On the other hand, when bentonite was applied as Trichoderma carrier, a stimulation of plant growth was also recorded, but in this case the increase in ascorbate recycling activities was accompanied by GST induction. Like ascorbate and POXs, GSTs have also been suggested to play a role in plant growth and development.$^{32}$

\section{CONCLUSIONS}

Our results concerning the use of Trichoderma-inoculated compost support the findings of Lopez-Mondejar et al., ${ }^{4}$ who demonstrated that combining citrus compost with $T$. harzianum T-78 is a viable alternative as a partial substitute for peat and that this treatment provides protection against Fusarium wilt during Cucumis melo L. 
seedling production. According to the data obtained in this study, the stimulation of the antioxidant capacity of a plant could be at least in part responsible for its tolerance to pathogen attack. Moreover, the measurement of antioxidant enzymes could be a potential marker of a plant's capacity to cope with environmental constraints. In this regard, antioxidant enzyme levels have been previously described as markers for identifying varieties of apricot that are resistant to Plum pox virus ${ }^{33}$ and as markers for salt stress tolerance in pea plants. ${ }^{34}$ An increase in ascorbate recycling enzymes can suggest higher reduced ASC content in melon plants. The combination of compost with Trichoderma can thus produce a sustainable increase in ASC content in melon plants and can lead to an improvement in the nutritional status of the plants and help them better respond to stress. Using compost-based growing media amended with $T$. harzianum T-78 therefore constitutes an environmentally friendly strategy for melon plant production.

\section{ACKNOWLEDGMENTS}

This work was supported by the FPU Programme of the Spanish Ministry of Education and the CYCIT AGL2010-21073 project from the Spanish Ministry of Economy and Competitivity. The study was also carried out as part of the Excellence Group, reference 04537/GERM/06, funded by the Seneca Foundation (Murcia, Spain). PDV acknowledges CSIC and the Spanish Ministry of Economy and Competitiveness for his “Ramon y Cajal” research contract, co-financed with FEDER funds. 


\section{REFERENCES}

1. Ros M, Hernández MT, Garcia C, Bernal A, Pascual JA, Biopesticide effect of green compost against Fusarium wilt on melon plants. J Appl Microbiol 98(4): 845-854 (2005).

2. Bernal-Vicente A, Ros M, Tittarelli F, Intrigliolo F, Pascual JA, Citrus compost and its water extract for cultivation of melon plants in greenhouse nurseries. Evaluation of nutriactive and biocontrol effects. Bioresour Technol 99: 8722-8728 (2008).

3. Harman GE, Howell CR, Viterbo A, Chet I, Lorito M, Trichoderma species: opportunistic, avirulent plant symbionts. Nat Rev Microbiol 2: 43-56 (2004).

4. Lopez-Mondejar R, Bernal-Vicente A, Ros M, Tittarelli F, Canali S, Intrigiolo F, Pascual JA, Utilisation of citrus compost-based growing media amended with Trichoderma harzianum T-78 in Cucumis melo L. seedling production. Bioresour Technol 101: 3718-3723 (2010).

5. Singh A, Srivastava S, Singh HB, Effect of substrates on growth and shelf life of Trichoderma harzianum and its use in biocontrol of diseases. Bioresour Technol 98: 470-473 (2007).

6. Diaz-Vivancos P, Clemente-Moreno MJ, Rubio M, Olmos E, Garcia JA, MartinezGomez P, Hernández JA, Alteration in the chloroplastic metabolism leads to ROS accumulation in pea plants in response to plum pox virus. J Exp Bot 59: 21472160 (2008).

7. Diaz-Vivancos P, Faize M, Barba-Espin G, Faize L, Petri C, Hernández JA, Burgos L, Ectopic expression of cytosolic superoxide dismutase and ascorbate peroxidase leads to salt stress tolerance in transgenic plums. Plant Biotech J 11: 976-985 (2013).

8. Jaspers P, Kangasjärvi J, Reactive oxygen species in abiotic stress signaling. Physiol Plant 138: 405-413 (2010).

9. Shoresh M, Harman GE, Mastouri F, Induced systemic resistance and plant responses to fungal biocontrol agents. Annu Rev Phytopathol 48: 1-23 (2010).

10. Tartoura KAH, Youssef SA, Stimulation of ROS-scavenging systems in squash (Cucurbita pepo L.) plants by compost supplementation under normal and low temperature conditions. Sci Hortic 130: 862-868 (2011).

11. Alguacil M, Caravaca F, Diaz-Vivancos P, Hernández JA, Roldan A, Effect of arbuscular mycorrhizae and induced drought stress on antioxidant enzyme and nitrate reductase activities in Juniperus oxycedrus L. grown in a composted sewage sludge-amended semi-arid soil. Plant Soil 279: 209-218 (2006).

12. Roldan A, Diaz-Vivancos P, Hernández JA, Carrasco L, Caravaca F, Superoxide dismutase and total peroxidase activities in relation to drought recovery 
performance of mycorrhizal shrub seedlings grown in an amended semiarid soil. $J$ Plant Physiol 165: 715-722 (2008).

13. Mastouri F, Björkman T, Harman GE, Trichoderma harzianum Enhances Antioxidant Defense of Tomato Seedlings and Resistance to Water Deficit. Mol Plant Microbe In 25: 1264-1271 (2012).

14. Bernal-Vicente A, Ros M, Pascual JA, Increased effectiveness of the Trichoderma harzianum isolate T-78 against Fusarium wilt on melon plants under nursery conditions. J Sci Food Agric 89: 827-833 (2009).

15. Bernal-Vicente A, Ros M, Pascual JA, Inoculation of Trichoderma harzianum during maturation of vineyard waste compost to control muskmelon fusarium wilt. Bioresources 7: 1948-1960 (2012).

16. Tittarelli F, Trinchera A, Intrigliolo F, Calabretta ML, De Simone C, Pierandrei F, Rea E, Production and utilisation of compost from citrus wastes of industrial processing, in Proceedings of the Fourth International Conference of Organic Recovery and Biological Treatment (ORBIT) Association on Biological Processing of Organics: Advances for a Sustainable Society. Perth (Australia), Part 2, pp. 818-826 (2003).

17. HabingWH, Jakoby WB, Assays for differentiation of glutathione S-transferases. Method Enzymol 77: 398-405 (1981).

18. Bradford MM, A rapid and sensitive method for the quantitation of microgram quantities of protein utilizing the principle of protein-dye binding. Anal Biochem 72: 248-254 (1976).

19. Lakhdar A, Iannelli MA, Debez A, Massacci A, Jedidi N, Abdelly C, Effect of municipal solid waste compost and sewage sludge use on wheat (Triticum durum): growth, heavy metal accumulation, and antioxidant activity. J Sci Food Agric 90: 965-971 (2010).

20. Hänsch R, Mendel RR, Physiological functions of mineral micronutrients $(\mathrm{Cu}, \mathrm{Zn}$, Mn, Fe, Ni, Mo, B, Cl). Curr Opin Plant Biol 12: 259-266 (2009).

21. Faize M, Burgos L, Faize L, Piqueras A, Nicolas E, Barba-Espin G, ClementeMoreno MJ, Alcobendas R, Artlip T, Hernández JA, Involvement of cytosolic ascorbate peroxidase and $\mathrm{Cu} / \mathrm{Zn}$-superoxide dismutase for improved tolerance against drought. J Exp Bot 62: 2599-2613 (2011).

22. Faize M, Burgos L, Faize L, Petri C, Barba-Espin G, Diaz-Vivancos P, ClementeMoreno MJ, Alburquerque N, Hernández JA, Modulation of tobacco bacterial disease resistance using cytosolic ascorbate peroxidase and $\mathrm{Cu}, \mathrm{Zn}$-superoxide dismutase. Plant Pathol 61: 858- 866 (2012).

23. Almagro L, Gómez Ros LV, Belchi-Navarro S, Bru R, Ros Barceló A, Pedreño MA, Class III peroxidases in plant defence reactions. J Exp Bot 60: 377-390 (2009). 
24. Edwards R, Dixon DP, Walbot V, Plant glutathione $S$-transferases: enzymes with multiple functions in sickness and in heath. Trends Plant Sci 5: 193-198 (2000).

25. Singh BN, Singh A, Singh SP, Singh HB, Trichoderma harzianum- mediated reprogramming of oxidative stress response in root apoplast of sunflower enhances defence against Rhizoctonia solani. Eur J Plant Pathol 131: 121-134 (2011).

26. Eltayeb AE, Kawano N, Badawi GH, Kaminaka H, Sanekata T, Morishima I, Shibahara T, Inanaga S, Tanaka K, Enhanced tolerance to ozone and drought stresses in transgenic tobacco overexpressing dehydroascorbate reductase in cytosol. Physiol Plant 127: 57-65 (2006).

27. Eltayeb AE, Kawano N, Badawi GH, Kaminaka H, Sanekata T, Shibahara T, Inanaga S, Tanaka K, Overexpression of monodehydroascorbate reductase in transgenic tobacco confers enhanced tolerance to ozone, salt and polyethylene glycol stresses. Planta 225: 1255-1264 (2007).

28. Gallo M, Esposito G, Ferracane R, Vinale F, Naviglio D, Beneficial effects of Trichoderma genus microbes on qualitative parameters of Brassica rapa L. subsp. sylvestris L. Janch. var. esculenta Hort. Eur Food Res Tech 236: 1063-1071 (2013).

29. Pastori GM, Kiddle G, Antoniw J, Bernard S, Veljovic-Jovanovic S, Verrier PJ, Noctor G, Foyer CH, Leaf Vitamin C Contents Modulate Plant Defense Transcripts and Regulate Genes That Control Development through Hormone Signaling. Plant Cell 15: 939-951 (2003).

30. Veljovic-Jovanovic SD, Pignocchi C, Noctor G, Foyer CH, Low ascorbic acid in the $v t c-1$ mutant of Arabidopsis is associated with decreased growth and intracellular redistribution of the antioxidant. Plant Physiol 127: 426-435 (2001).

31. De Gara L, Ascorbate metabolism and plant growth-from germination to cell death, in Vitamin C: its Function and Biochemistry in Animals and Plants, ed. by BIOS Scientific Publishers, Oxford, pp. 83-95 (2003).

32. Gong H, Jiao Y, Hu WW, Pua EC, Expression of glutathione-S-transferase and its role in plant growth and development in vivo and shoot morphogenesis in vitro. Plant Mol Biol 57: 53-66 (2005).

33. Hernández JA, Diaz-Vivancos P, Rubio M, Olmos E, Ros-Barceló A, MartínezGómez P, Long-term PPV infection produces an oxidative stress in a susceptible apricot cultivar but not in a resistant cultivar. Physiol Plant 126: 140-152 (2006).

34. Hernández JA, Jiménez A, Mullineaux PM, Sevilla F, Tolerance of pea (Pisum sativum L.) to long-term salt stress is associated with induction of antioxidant defences. Plant Cell Environ 23: 853-862 (2000). 


\section{Tables}

Table 1. Physiochemical and chemical characteristics of the composts and peat used. EC, electrical conductivity.

\begin{tabular}{|c|c|c|c|}
\hline & Peat & C1 & $\mathrm{C} 2$ \\
\hline pH & 6.34 & 6.71 & 8.5 \\
\hline $\operatorname{EC~}\left(\mathrm{mS} \mathrm{cm}^{-1}\right)$ & 0.51 & 1.89 & 1.78 \\
\hline N (\%) & 0.97 & 0.89 & 2.5 \\
\hline P (\%) & 0.7 & 0.06 & 0.7 \\
\hline K (\%) & 0.6 & 0.69 & 0.7 \\
\hline $\mathrm{C} / \mathrm{N}$ & 50 & 43.58 & 15 \\
\hline
\end{tabular}

Table 2. F-values from two-way ANOVA (T, Trichoderma; S, substrate) for fresh weight and antioxidant enzymes in the leaves of melon plants. FW, fresh weight; APX, ascorbate peroxidase; MDHAR, monodehydroascorbate reductase; DHAR, dehydroascorbate reductase; SOD, superoxide dismutase; POX, peroxidase; GST, glutathione $S$-transferase; CAT, catalase.

\begin{tabular}{ccccccccc}
\hline & FW & APX & MDHAR & DHAR & SOD & POX & GST & CAT \\
\hline $\mathbf{T}$ & $18.4^{* * *}$ & $9.0^{* *}$ & $57.0^{* * *}$ & $10.5^{* *}$ & $4.9^{*}$ & 0.1 & $5.7^{*}$ & 0.6 \\
$\mathbf{S}$ & $20.7^{* * *}$ & $105.5^{* * *}$ & $8.5^{* *}$ & $59.7^{* * *}$ & $9.2^{* *}$ & $918.3^{* * *}$ & $14.6^{* * *}$ & 0.9 \\
$\mathbf{T} \mathbf{x} \mathrm{S}$ & $12.4^{* * *}$ & $38.2^{* * *}$ & $94.4^{* * *}$ & $40.6^{* * *}$ & 3.6 & $95.0^{* * *}$ & $18.3^{* * *}$ & 0.9 \\
\hline
\end{tabular}

F-values significant at $99.9 \%\left({ }^{* * *}\right), 99 \%(* *)$, or $95 \%(*)$ levels of probability. 


\section{Figures legends:}

Fig. 1. Fresh weight (FW) of melon plants grown in the different organic substrates analyzed in presence and absence of T. harzianum T-78. Bento, bentonite supplemented peat; C1, compost C1; C2, compost C2. Different letters indicate statistical significance according to Tukey's test $(\mathrm{P}<0.05)$.

Fig. 2. Ascorbate-glutathione cycle enzyme (A, APX; B, MDHAR; C, DHAR) activities in leaves of melon plants grown in the different organic substrates analyzed in the presence and absence of T. harzianum T-78. Bento, bentonite supplemented peat; C1, compost C1; C2, compost C2. Different letters indicate statistical significance according to Tukey's test $(\mathrm{P}<0.05)$.

Fig. 3. Antioxidant enzyme (A, SOD; B, POX; C, GST) activities in leaves of melon plants grown in the different organic substrates analyzed in the presence and absence of T. harzianum T-78. Bento, bentonite supplemented peat; C1, compost C1; C2, compost C2. Different letters indicate statistical significance according to Tukey's test $(\mathrm{P}<$ $0.05)$. 